\title{
Bound state of solution of Dirac-Coulomb problem with spatially dependent mass
}

\author{
Eser Olğar ${ }^{1}$, Hayder M. Dhahir ${ }^{1}$, H. Mutaf ${ }^{1}$ \\ Gaziantep University, Engineering of Physics Department, Gaziantep/TURKEY*
}

(Dated: August 13, 2018)

\begin{abstract}
The bound state solution of Coulomb Potentials in Dirac equation is calculated for position dependent mass function $M(r)$ within the framework asymptotic iteration method (AIM). The eigenfunctions are derived in terms of hypergeometric function using function generator equation of AIM.
\end{abstract}

PACS numbers: 03.65.Ge; 03.65.Fd

Keywords: Dirac equation, Coulomb Potentials, position dependent mass, asymptotic iteration method

*Electronic address: olgar@gantep.edu.tr, rg_s3@yahoo.com, hmutaf@gantep.edu.tr 


\section{INTRODUCTION}

The solution of the relativistic Dirac equation for quantum mechanical systems in both case of spatially dependent mass and constant mass plays an important role in many branches of physics [1-15]. Since the importance of investigation of the Dirac equation with position

dependent mass, there has been increased a great interest on it [5-15]. Dirac equation with position-dependent mass for solvable potentials has been addressed by a number of different methods [16-23]. Besides these methods, recently Ciftci et al [24-26] proposed an asymptotic iteration method (AIM) which draws the attention of a many researchers for relativistic equations [27-33].

This method has the advantages of obtaining the solution of eigenvalue problems without solving directly the differential equation. Dirac equation with first order differential equation form with two dimensional (2D) Coulomb potential for constant mass [34 40] and for spatially dependent mass [41] has been solved by using AIM. M. Hamzavi and collaborators 41] have been considered the Coulomb potential including a Coulomb-like tensor potential under pseudospin symmetry limit.

The main purpose of this study is to solve the bound state sollution of Coulomb potential for position dependent mass of Dirac equation without Coulomb-like tensor potential by considering the relation between vector and scalar potential as $S(r)=V(r)(b-1)$ where $b$ is arbitrary parameter [30, 31]. The second section represents the formalism of Dirac equation with position dependent mass. The asymptotic iteration method is introduced in Section 3. The calculation of eigenvalues and eigenfunctions of Coulomb potential are outlined in the subsequent section. The last section devotes to conclusion.

\section{FORMALISM OF THE DIRAC EQUATION}

The Dirac equation for a central field in 3-dimensions is written for spherically symmetric vector $V(r)$ and $S(r)$ spherically symmetric scalar potential by using the parameters $\hbar=$ $c=1$ as

$$
E_{n l} \Psi=\left[\sum_{j=1}^{3} \alpha_{j} p_{j}+\beta(m+S(r))+V(r)\right] \Psi
$$

where $m$ is the mass of the particle, $S(r)$ is a spherically symmetric scalar potential, $V(r)$ is a spherically symmetric vector potential, $\alpha$ and $\beta$ are the usual Dirac matrices satisfying 
anticommutation relations, and $E_{n l}$ is the corresponding eigenvalues. After some algebraic calculations, one obtains the following first-order linear coupled differential equations (11) .

Without any approximation, the Dirac equation for a central field in spherical coordinates can be separated into the variables. Thus, it has mean the eigenfunction of the orbital and spin angular momentum can be found as

$$
\begin{aligned}
\frac{d F_{n k}(r)}{d r}+\frac{k}{r} F_{n k}(r) & =\left(E_{n l}+M(r)-V(r)+S(r)\right) Q_{n k}(r) \\
\frac{d Q_{n k}(r)}{d r}-\frac{k}{r} Q_{n k}(r) & =-\left(E_{n l}-M(r)-V(r)-S(r)\right) F_{n k}(r)
\end{aligned}
$$

where $k=-(l+1)$ for the total angular momentum $j=l+1 / 2$, and $l$ is angular momentum quantum number. $F_{n k}(r)$ and $Q_{n k}(r)$ are the radial wave function of the upper and the lower-spinor components respectively, and the general form of two second-order differential equations for corresponding eigenfunctions are obtain by eliminating wave function $F_{n k}(r)$ in Eq. (2) and $Q_{n k}(r)$ in Eq. (3) we get

$$
\left[\frac{d^{2}}{d r^{2}}-\frac{k(k+1)}{r^{2}}\right] F_{n k}(r)-\frac{\left(\frac{d M(r)}{d r}-\frac{d \Delta(r)}{d r}\right)\left(\frac{d}{d r}+\frac{k}{r}\right) F_{n k}(r)}{M(r)+E_{n l}-\Delta(r)}=\left[\left(M(r)+E_{n l}-\Delta(r)\right)\left(M(r)-E_{n l}+\sum(r)\right)\right] F_{n k}(r)
$$

and

$$
\left[\frac{d^{2}}{d r^{2}}-\frac{k(k-1)}{r^{2}}\right] Q_{n k}(r)-\frac{\left(\frac{d M(r)}{d r}+\frac{d \Delta(r)}{d r}\right)\left(\frac{d}{d r}-\frac{k}{r}\right) Q_{n k}(r)}{M(r)-E_{n l}+\Delta(r)}=\left[\left(M(r)+E_{n l}-\Delta(r)\right)\left(M(r)-E_{n l}+\sum(r)\right)\right] Q_{n k}(r)
$$

where

$$
\sum(r)=V(r)+S(r), \text { and } \Delta(r)=V(r)-S(r) .
$$

We use the relationship between scalar and vector potentials,

$$
S(r)=V(r)(b-1), b \geq 0
$$

to define $\sum(r)$ and $\Delta(r)$. In this general description of scalar potential, by choosing $b$ parameter 0,1 , and 2 the scalar potential leads to case of $S(r)=-V(r), S(r)=0$ (purely vector potential), and $S(r)=V(r)$ respectively. The other choices of $b$ leads to the required condition for the case of $S(r)>V(r)$. This transformation yields to

$$
\sum(r)=b V(r), \text { and } \Delta(r)=(2-b) V(r) .
$$




\section{ASYMPTOTIC ITERATION METHOD}

The AIM is proposed to solve the second-order linear differential equation in the form of

$$
y^{\prime \prime}=\lambda_{0}(x) y^{\prime}+s_{0}(x) y
$$

where the functions $\lambda_{0}(x)$ and $s_{0}(x)$ are differentiable with $\lambda_{0}(x) \neq 0$. Shortly, the solution of any differential equation can be written in form of Eq. (6) has a general solution within the framework AIM as

$$
y(x)=\exp \left(-\int \alpha d t\right)\left[C_{2}+C_{1} \int \exp \left(\int\left(\lambda_{0}(\tau)+2 \alpha(\tau)\right) d \tau\right) d t\right]
$$

where $C_{i}$ are integral constants. The arbitrary functions for the limit of $n$ are

$$
\begin{aligned}
& \lambda_{n}(x)=\lambda_{n-1}^{\prime}(x)+s_{n-1}(x)+\lambda_{0}(x) \lambda_{n-1}(x) \\
& s_{n}(x)=s_{n-1}^{\prime}(x)+s_{0}(x) \lambda_{n-1}(x) .
\end{aligned}
$$

with asymptotic expression

$$
\frac{s_{n}(x)}{\lambda_{n}(x)}=\frac{s_{n-1}(x)}{\lambda_{n-1}(x)}=\alpha(x)
$$

And the termination condition is in the form of

$$
\Delta_{k}(x)=\left|\begin{array}{cc}
s_{n}(x) & \lambda_{n}(x) \\
s_{n-1}(x) & \lambda_{n-1}(x)
\end{array}\right|=\lambda_{n-1}(x) s_{n}(x)-\lambda_{n}(x) s_{n-1}(x), \quad k=1,2,3, \ldots
$$

which gives the solution of physical systems.

\section{SOLUTION OF THE DIRAC - COULOMB PROBLEM}

The Dirac-Coulomb potential is considered by proposing the coulomb like vector potential and scalar potential as

$$
V(r)=\frac{V_{0}}{r}, \text { yields to } S(r)=\frac{V_{0}(b-1)}{r} .
$$

The Eq. (4) can not be solved analytically because of the effect of the last term $\left(\frac{d M(r)}{d r}-\frac{d \Delta(r)}{d r}\right)$. Therefore, we calculate the required mass function that satisfies the equality $\left(\frac{d M(r)}{d r}-\frac{d \Delta(r)}{d r}=\right.$ 
0) to eliminate this effect. Thus, using this equality condition, the mass function is obtained as the following function

$$
M(r)=\frac{(2-b) V_{0}}{r}+m_{0}
$$

where $m_{0}$ is the rest mass of the fermionic particle and $(2-b) V_{0}$ is the perturbed mass [42]. By substituting the potential functions in Eq. (8) and variable mass function in Eq. (9) into Eq. (4), we get

$$
\frac{-k(k+1)}{r^{2}} F_{n k}(r)-\left(E_{n l}+m_{0}\right)\left(-E_{n l}+\frac{2 V_{0}\left(E_{n l}+m_{0}\right)}{r}+m_{0}\right) F_{n k}(r)+\frac{d^{2}}{d r^{2}} F_{n k}(r)=0
$$

At this point, if the following notations are made

$$
-E_{n l}^{2}+m_{0}^{2}=\varepsilon_{n l}^{2}, k(k+1)=A(A+1),-2\left(E_{n l}+m_{0}\right) V_{0}=B
$$

the eigenvalues equation transforms to

$$
\left(-\varepsilon_{n l}^{2}-\frac{A(A+1)}{r^{2}}+\frac{B}{r}\right) F_{n k}(r)+\frac{d^{2} F_{n k}(r)}{d r^{2}}=0
$$

Propose the wavefunction by using AIM as

$$
F_{n k}(r)=r^{A+1} \exp \left[-\varepsilon_{n l}\right] \chi(r)
$$

Then, by substituting this wavefunction into Eq. (12), we obtain

$$
\left(B-2(1+A) \varepsilon_{n l}\right) \chi(r)+2\left(1+A-\varepsilon_{n l} r\right) \chi^{\prime}(r)+r \chi^{\prime \prime}(r)=0 .
$$

Thus, $\chi^{\prime \prime}(r)$ becomes

$$
\chi^{\prime \prime}[r]=\frac{-2\left(1+A-\varepsilon_{n l} r\right)}{r} \chi^{\prime}[r]+\frac{-B+2(1+A) \varepsilon_{n l}}{r} \chi[r]
$$


By comparing Eq. (14) with the second-order differential equation of Eq. (66), we get the arbitrary functions $\lambda_{0}(r)$ and $s_{0}(r)$. The values of arbitrary functions are

$$
\begin{aligned}
& \lambda_{0}(r)=2\left[\frac{\varepsilon_{n l} r-A-1}{r}\right] \\
& s_{0}(r)=\frac{2(A+1) \varepsilon_{n l}-B}{r} \\
& \lambda_{1}(r)=\frac{4 \varepsilon_{n l}^{2} r^{2}-6 A \varepsilon_{n l} r-6 \varepsilon_{n l} r-B u+4 A^{2}+10 A+6}{u^{2}}, \\
& s_{1}(r)=\frac{\left(B-2(A+1) \varepsilon_{n l}\right)\left(2 A-2 \varepsilon_{n l} r+3\right)}{r^{2}}
\end{aligned}
$$

By using termination condition for energy, we get

$$
\begin{aligned}
& \frac{s_{0}(r)}{\lambda_{0}(r)}=\frac{s_{1}(r)}{\lambda_{1}(r)} \Longrightarrow \varepsilon_{0 l}=\frac{B}{2(A+1)} \\
& \frac{s_{1}(r)}{\lambda_{1}(r)}=\frac{s_{2}(r)}{\lambda_{2}(r)} \Longrightarrow \varepsilon_{1 l}=\frac{B}{2(A+2)} \\
& \frac{s_{2}(r)}{\lambda_{2}(r)}=\frac{s_{3}(r)}{\lambda_{3}(r)} \Longrightarrow \varepsilon_{2 l}=\frac{B}{2(A+3)}
\end{aligned}
$$

The general formula of $\xi$ for $n$ values can be written as

$$
\varepsilon_{n l}=\frac{B}{2(n+A+1)}, \quad n=0,1,2, \ldots \ldots
$$

The eigenvalues in Eq. (16) is transformed into the form of $E_{n}$ by the definition of the parameter $\varepsilon_{n l}$ in Eq. (11)

$$
E_{n l}^{2}=m_{0}^{2}-\left(\frac{B}{2(n+A+1)}\right)^{2}
$$

The corresponding energy eigenfunctions can be found by using the generator

$$
\chi(r)=\exp \left(-\int^{r} \frac{s_{k}(r)}{\lambda_{k}(r)} d r\right.
$$

By applying the function generator, the $f_{n}(r)$ functions can be written in series expansion by hypergeometric functions with constant $(B+n+1)^{n}$ and $\prod_{k=0}^{(n-1)}(B+2+k)$. By generalizing these expansions, we get

$$
\chi(r)=(B+n+1)^{n}\left[\prod_{0}^{n-1}(2 B+2+k)\right] x_{1} F_{1}\left(-n, 2 B+2 ; 2 \varepsilon_{n l} r\right)
$$


We write the upper spinor component of the radial wave function as

$$
F_{n k}(r)=r^{A+1} e^{\left(-\varepsilon_{n l} r\right)}(B+n+1)^{n}\left[\prod_{0}^{n-1}(2 B+2+k)\right] x_{1} F_{1}\left(-n, 2 B+2 ; 2 \varepsilon_{n l} r\right)
$$

The lower spinor wave function can be obtained in a similar algebraic calculation. The mass function for lower spinor is calculated as $M(r)=\frac{(b-2) V_{o}}{r}+m_{0}$. After all same algebraic procedure, we get the same results for eigenfunctions and eigenvalues with different parameters as

$$
G_{n k}(r)=r^{A+1} e^{\left(-\varepsilon_{n l} r\right)}(B+n+1)^{n}\left[\prod_{0}^{n-1}(2 B+2+k)\right] x_{1} F_{1}\left(-n, 2 B+2 ; 2 \varepsilon_{n l} r\right)
$$

where

$$
\begin{aligned}
E_{n l}^{2}-m_{0}^{2} & =-\varepsilon_{n l}^{2}, \\
k(k-1)+4 V_{0}^{2}\left(b^{2}-3 b+2\right) & =A(A+1), \\
2\left(E_{n l}+(2 b-3) m_{0}\right) V_{0} & =B
\end{aligned}
$$

These eigenfunctions and eigenvalues obey the results in [41] after mapping the corresponding parameters with those of in [41]].

\section{CONCLUSION}

The spectrum of position dependent mass Dirac equation for Coulomb potential is obtained within the framework of AIM method without solving the differential equation. The mass function is considered as in the form of function satisfying the equality condition, $\frac{d M(r)}{d r}-\frac{d V(r)}{d r}=0$. When the vector potential $V(r)$ is considered equal (states $b=2$ ) to the spherical Scalar potential, the mass function is reduced to constant mass situation. In upper spinor wavefunction, if $b>2(S(r)>V(r))$, the perturbed mass term results a negatively effect in $M(r)$. But in lower spinor wavefunction, this condition yields to a positively effect in $M(r)$. Therefore, by adjusting the parameter $b$, the bound-state solutions for spinor wavefunctions is calculated by applying AIM and compared with corresponding results in [41]. 


\section{ACKNOWLEDGEMENT}

This research was supported by the Research Fund of Gaziantep University (BAP) and the Scientific and Technological Research Council of TURKEY (TÜBITAK).

[1] W. Griner, Relativistic Quantum Mechanics Wave Equation (3rd edition, Freankfert: Germen, 1987)

[2] A. D. Saavedra, F. Boronat, J. Polls, and A. Fabrocini, Phys. Rev. B 50, 4248 (1994)

[3] T. Gora, and F. Williams, Phys. Rev. 177, 1179 (1969)

[4] A. D. Alhaidari, Phys. Rev. A 75, 042707 (2007)

[5] A. D. Alhaidari, H. Bahlouli, A. Al Hasan, and M.S. Abdelmonem, Phys. Rev. A 75, 062711 (2007)

[6] A. D. Alhaidari, Phys. Lett .A 322, 72 (2004)

[7] O. Panella, S. Biondini, and A. Arda, J. Phys. A Math. Theor. 43, 325302 (2010)

[8] A. De Souza Dutra, and C.S. Jia, Phys. Lett. 352, 484 (2010)

[9] I. O. Vakarchuk, J. Phy. A Math. Gen. 38, 4727 (2005)

[10] S. M. Ikhadair, and R. Sever, Appl. Math. Comput. 216, 911 (2010)

[11] C. S. Jia, T. Chen, and L.G. Cui, Phys. Lett. A 373, 1621 (2009)

[12] C. S. Jia, and A. de Souza Dutra, Ann. Phys. 323, 566 (2008)

[13] L. Dekar, L. Chetouani, T.F. Hammann, J.Math. Phys. 39, 2551 (1998)

[14] X. L. Peng, J.Y. Liu, and C.S. Jia, Phys. Lett. A 352, 478 (2006)

[15] D. Agboola, Preprint math-ph/arxiv: 1011, 2368v1.

[16] J. Wu., and Y. Alhassid, J. Math. Phys. 31, 557 (1990)

[17] R.A. Swainson, and G.W.F. Drake, J. Phys. A: Math. Gen. 24, 79 (1991)

[18] G. Chen, Phys. Lett. A 326, 55 (2004)

[19] L. Infeld and T.E. Hull, Rev. Mod. Phys. 23, 21 (1951)

[20] R.P. Feynman, and A.R. Hibbs, Quantum Mechanics and Path Integrals, (New York. McGrawHill, 1965).

[21] C. Grosche, J. Phys. A. Math. Gen. 28, 5889 (1995)

[22] E. Witten, Nucl. Phys. B 188, 513 (1981) 
[23] A. Comtet, A. Bandrank A and D.K. Campbell, Phys. Lett. B 150, 159 (1985)

[24] H. Ciftci, R.L. Hall, N. and Saad, J. Phys. A: Math. Gen. 36, 11807 (2003)

[25] H. Ciftci, R.L. Hall, N. and Saad, J. Phys. A: Math. Gen. 38, 1147 (2005).

[26] F. M. Fernandez, J. Phys. A. Math. Gen. 37, 617332 (2004).

[27] T. Barakat, K. Abodayeh, and A. Mukheimer, J. Phys. A. Math. Gen. 38, 1299 (2005)

[28] T. Barakat, Phys. Lett. A 334, 411 (2005).

[29] E. Olğar, R. Koç, and H. Tütüncüler, Chin. Phys. Lett. 23, 539 (2006);

[30] E. Olğar, Chin. Phys. Lett. 26, 020302 (2009)

[31] E. Olğar and H. Mutaf, Commun. Theor. Phys. (Beijing, China) 53, 1043 (2010)

[32] E. Olğar, Chin. Phys. Lett. 25, 1939 (2008)

[33] E. Olğar, R. Koç, and H. Tütüncüler, Phys. Scr. 78, 015011 (2008).

[34] R. E. Moss, Am. J. Phys. 55, 397 (1987).

[35] H. Galic, Am. J., Phys. 56, 312 (1988) .

[36] S. H. Dong and Z. Q. Ma., Phys. Lett. 312, 78 (2003).

[37] D. Agboola, Int. J, Quantum Chem. 112, 1029 (2012)

[38] D. Agboola, Few Body Syst. 52, 31 (2012)

[39] D. Agboola, Pramana, Phys. 76 (6), 875 (2011).

[40] G. Esposito, and P. Santorelli, J. Phys. A. Gen. 32, 5643 (1999).

[41] M. Hamzavi, A.A. Rajabi, and H. Hassanabadi, Phys. Let. A 374, 4303 (2010)

[42] S. M. Ikhdair, R. Sever, Appl. Math. Phys. 316, 545 (2010). 